\title{
Percepción de la violencia escolar en el último ciclo de educación primaria
}

\section{Perception of violence in the last cycle of elementary school}

\author{
Luisa Fernanda Sapién Zúñiga ${ }^{1}$ \\ Priscila Ledezma Rivas ${ }^{2}$ \\ José Guadalupe Ramos Trevizo ${ }^{3}$
}

\begin{abstract}
Resumen
El estudio presenta los resultados de la aplicación del Cuestionario de Violencia Escolar (CUVE) que permite a través de una escala tipo Likert medir las percepciones que tienen los alumnos respecto a la violencia en las escuelas. Se eligieron como sujetos de la investigación a niños del último ciclo escolar de una escuela primaria periférica en la ciudad de Chihuahua cuyas edades oscilan entre los 10 y 13 años de edad y que por las condiciones de aplicación del instrumento permiten validar sus resultados. El instrumento arroja que la violencia verbal es una de las formas de violencia que mayormente se lleva a cabo entre los estudiantes que participaron de la investigación, a través de ello se comprueba que las formas de agresión no solamente apuntan hacia lastimar físicamente al otro, sino a otras maneras que también son sentidas por los alumnos. Se pretende que el insumo que
\end{abstract}

1 Luisa Fernanda Sapién Zúñiga. Institución Benemérita y Centenaria Escuela Normal del Estado de Chihuahua Profesor Luis Urías Belderráin, México. Licenciatura en Educación Preescolar. Correo electrónico: feysapien@gmail.com ID: http://orcid.org/0000-0003-1600-4439

2 Priscila Ledezma Rivas. Institución Benemérita y Centenaria Escuela Normal del Estado de Chihuahua Profesor Luis Urías Belderráin, México. Licenciatura en Educación Primaria. Correo electrónico: priscilaledezmarivas@gmail.com ID: http://orcid.org/0000-0001-6949-8829

${ }^{3}$ José Guadalupe Ramos Trevizo. Docente en la Institución Benemérita y Centenaria Escuela Normal del Estado de Chihuahua Profesor Luis Urías Belderráin, México. Maestro en Historia por la Universidad Autónoma de Ciudad Juárez. Correo electrónico: j.ramos@ibycenech.edu.mx

ID: http://orcid.org/0000-0003-2054-2061 
RECIE. Revista Electrónica Científica de Investigación Educativa

Vol. 4, núm. 2, enero-diciembre 2019, pp. 1349-1360.

genera el estudio sirva para la toma de decisiones que permitan mejorar la convivencia dentro del centro escolar.

\title{
Palabras clave
}

Violencia escolar, educación primaria, percepción, cuestionario.

\begin{abstract}
The study presents the results of the questionnaire of school violence (CUVE) that allows through a scale Likert-type measure perceptions having students regarding violence in schools. Were chosen as subjects of research to children of the last school year from a peripheral elementary school in the city of Chihuahua, whose ages range from 10 to 13 years of age and that the conditions of application of the instrument allow to validate their results. The instrument shows that verbal violence is one of the forms of violence that mostly takes place among the students who participated in the research, through this checks to see that the forms of aggression not only point to hurt physically to the other, but to other ways that are also felt by students. It is intended that the input that generates the study will serve to make decisions that improve the coexistence within the school.
\end{abstract}

\section{Keywords}

School violence, elementary school, perception, questionnaire.

\section{Introducción}

En el informe de la Secretaría de Salud (SS) 2006 afirma que México se encuentra en el grupo de naciones más violentas del mundo, en relación con otros países latino americanos, México se encuentra en el sexto lugar, y como tal el ambiente en el país tiene impacto en los niños y niñas. La imposición de maltrato verbal o castigo físico, aunque puedan ser indefensos o menos visibles y menos dramáticos, implican dosis cotidianas de dolor que causan severos daños y disminuyen la posibilidad de una vida plena, sana y libre.

Pareciera que lo anterior permea hacia las aulas donde los niños y jóvenes llevan a cabo su vida académica toda vez que es posible identificar que los estudiantes tienen formas distintas de relacionarse y asuntos como el acoso escolar entre ellos, la violencia verbal y las agresiones físicas llegan incluso a ser noticia.

\section{Origen del término violencia}

La palabra violencia procede de la unión del latín vis- (fuerza) con el sufijo lentus (contínuo), cuya conjunción 'violentia'vendría a significar "uso 
continuado de la fuerza ", y cuya forma verbal 'violare 'explícitamente significaba "agredir ". Término recuperado del Diccionario de la Real Academia Española (RAE, 2017).

Desde tiempos antiguos, la violencia ha estado presente en todos los ámbitos de la sociedad, sus causas son muy diversas, es por eso que el término es catalogado como un fenómeno multicausal. El ser humano es desde su nacimiento una criatura agresiva es decir que posee un potencial innato, en el cual existe un sustrato psicológico que suscita sentimientos subjetivos de ira, así como cambios físicos que preparan al ser para la lucha (Cerezo, 2009).

\section{Definición de la violencia}

La OMS (Organización Mundial de la Salud, 1996) define violencia como:

El uso deliberado de la fuerza física o el poder, ya sea en grado de amenaza o efectivo, contra uno mismo, otra persona o un grupo o comunidad, que cause o tenga muchas probabilidades de causar lesiones, muerte, daños psicológicos, trastornos del desarrollo o privaciones.

La definición que presenta la OMS es considerada como las de mayor uso, sin embargo, el término se presta a ser una cuestión de apreciación de quienes lo manejan. Muchas veces la propia cultura es la que determina aquellas conductas aceptables e inaceptables, y esto puede someterse a una continua modificación a medida que los valores y las normas sociales evolucionan.

\section{Tipología de violencia}

La violencia puede presentar diversos tipos de manifestación, entre las que destacan se consideran las de tipo físico y verbal. En 1996, la Organización Mundial de la Salud declara la violencia como un importante problema de salud pública en todo el mundo, por lo tanto, evidenció la necesidad de elaborar una tipología de la violencia para caracterizar los diferentes tipos y los vínculos entre ellos.

La clasificación propuesta divide la violencia en 3 categorías generales, según las características del que comete el acto. Violencia Autoinflingida; violencia interpersonal y violencia colectiva (OMS, 2003, p. 19) (fig. 1) Estas tres categorías generales se subdividen a su vez para reflejar tipos de violencia más específicos.

\section{Violencia autoinfligida}

La violencia autoinfligida comprende el comportamiento suicida y autolesiones. El primero incluye pensamientos suicidas, intentos de suicidio 
RECIE. Revista Electrónica Científica de Investigación Educativa Vol. 4, núm. 2, enero-diciembre 2019, pp. 1349-1360.

también llamados parasuicidio. Por contraposición, las autolesiones incluyen actos como la automutilación (Krug et al, 2003, p. 26).

El suicidio representa la forma más extrema de la violencia autoinflingida, sabemos que es difícil que esta se presente en un niño de edad preescolar ya que comúnmente se da en adolescentes y adultos.

Violencia interpersonal

La violencia interpersonal se caracteriza por el comportamiento deliberado de la fuerza, esta puede llevar a sus miembros a causarse daños físicos o psicológicos cuando se dan amenazas u ofensas. La violencia interpersonal se divide en dos subcategorías:

- $\quad$ Violencia familiar o de pareja: Se produce sobre todo entre los miembros de la familia o en una relación sentimental, y que, por lo general, sucede en el hogar.

- Violencia comunitaria: Se produce entre personas que no guardan parentesco y que pueden conocerse o no, sucede por lo general fuera del hogar (Krug et al, 2003, pág. 26).

\section{Violencia colectiva}

Las subcategorías de la violencia colectiva indican los posibles motivos de la violencia cometida por grupos más grandes de individuos o por el Estado.

- La violencia social incluye, los actos delictivos de odio cometidos por grupos organizados, las acciones terroristas y la violencia de masas.

- La violencia cultural da cierta legitimidad al uso del arte, la religión, la ciencia, los derechos, las ideologías, los medios de comunicación.

- La violencia política incluye la guerra y otros conflictos violentos afines, la violencia del Estado y actos similares.

- La violencia económica comprende los ataques por parte de grupos más grandes motivados por el afán de lucro económico, como negar el acceso a servicios esenciales o crear división económica y fragmentación (Krug et al, 2003, pág.27). 


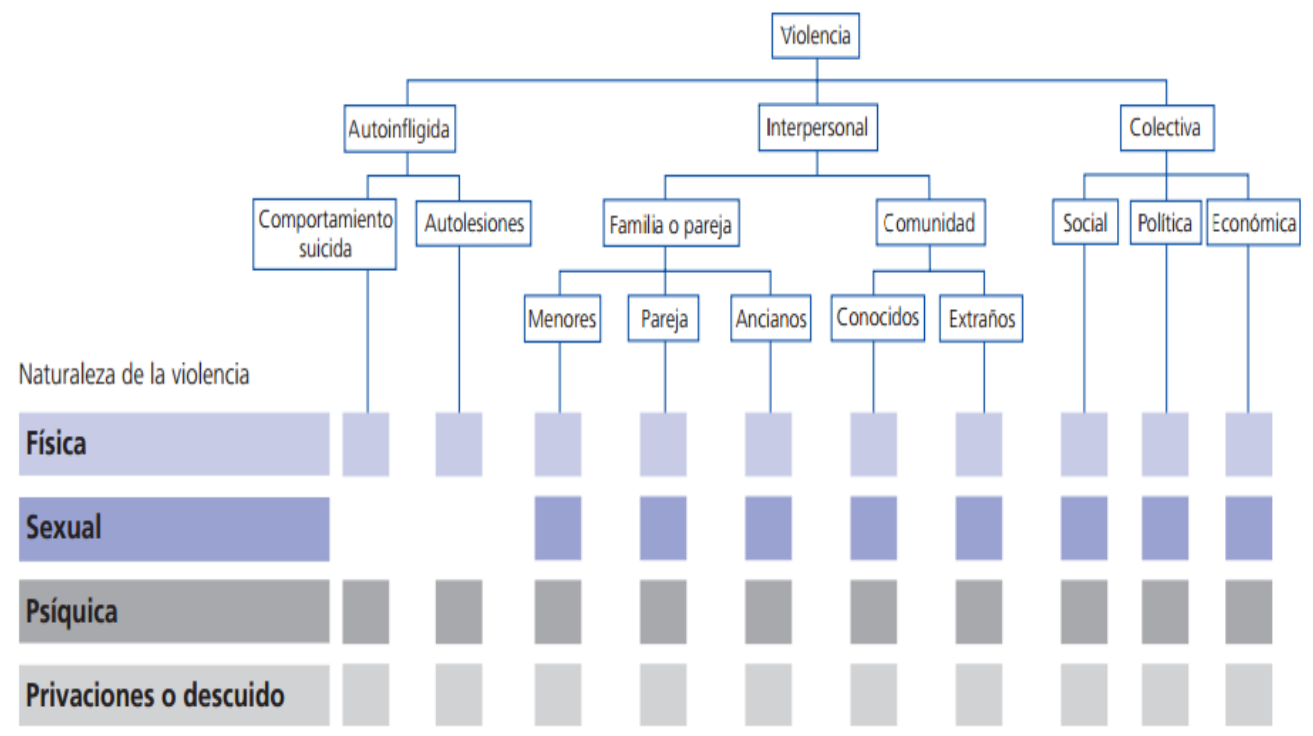

Figura 1. Clasificación de la violencia.

*Fuente: Tomada del Informe mundial de violencia y de la salud 2003.

\section{Acoso escolar o bullying}

La violencia escolar como término acuñado, aparece en la década de los cincuenta en Estados Unidos de América. Desde el inicio científico, las primeras referencias a estas situaciones se expresan como mobbing, entendido como la agresión de unos alumnos contra otros (Heinemann, 1969), siendo considerado por muchos autores como la antesala del bullying. Dicho término mobbing, tomado de Lorenz (1973).

El blog del arco atlántico (2005) se basa en la afirmación de Dan Olweus padre del término bullying para afirmar que:

El fenómeno del bullying ha existido desde hace mucho tiempo. Al comenzar su investigación se encontró con quienes argumentaban que la violencia era algo natural y, por tanto, necesario en la escuela, como una medida de preparación para la vida adulta. Esta concepción fue transformada y lo que se consideraba como natural pasó a ser un problema social.

Las agresiones que se manifiestan no necesariamente son físicas, también se encuentran las agresiones verbales las cuales son mucho más comunes, desde un insulto, una amenazas, rumores, apodos y especulaciones que tratan de afectar en su autoestima. Para que este acto se lleve a cabo es necesario que se presente un agresor y una víctima los cuales son los personajes protagonistas de la violencia. 
RECIE. Revista Electrónica Científica de Investigación Educativa Vol. 4, núm. 2, enero-diciembre 2019, pp. 1349-1360.

En primera instancia tenemos a un agresor o bullies el cual suele actuar motivado por el deseo de poder, de intimidar, dominar, sentirse superior a sus semejantes, entre otras, aunque en ocasiones se reconoce que están motivados por simple diversión. Por consiguiente, se encuentra la víctima la cual es reconocida por ser una persona indefensa, con autoestima baja, sin posibilidad de respuesta ni apoyos. La mayoría de las víctimas se caracteriza por presentar sumisión y pasividad ante su agresor, es decir, por mostrar retraimiento ante la situación de violencia (Schwartz, Proctor y Chien, 2001). Interesa por lo tanto saber que sucede en las instituciones de educación básica, principalmente en el tercer ciclo de educación primaria, es decir, en los grupos de quinto y sexto grado donde se concentra la mayor parte de alumnos que entran en la pre adolescencia o pubertad y donde la población permite el uso de un instrumento validado para medir la percepción de la violencia escolar, es decir el Cuestionario de Violencia Escolar (CUVE).

\section{Ruta metodológica}

El objetivo primordial de la investigación es conocer cuáles son los tipos de violencia que mayormente se presentan en grupos de quinto y sexto grado de una escuela primaria en la ciudad de Chihuahua. Además, se busca medir la percepción de violencia escolar en los alumnos y finalmente generar insumos de información para futuras investigaciones.

Se optó por utilizar un enfoque cuantitativo, inspirado en el positivismo.

Explica Monje 82011, p.11) que es utilizado en las ciencias sociales ya que, "su propósito es buscar explicación a los fenómenos estableciendo regularidades en los mismos" explicando el comportamiento social. Al elegir este enfoque, se establece un parámetro de evaluación, en los que los test aplicados cuentan con cierta numeración para facilitar la interpretación de datos.

Se utilizó el CUVE (Cuestionario de Violencia Escolar) que es una prueba que ayuda a comprobar cuáles son los grados de violencia dirigida en la escuela, ya sea infringida en el mismo alumno, siendo víctimas o victimarios. Esta corresponde a una serie de situaciones, las cuales evalúan cual es la percepción de violencia en el alumno.

Este instrumento mide la percepción de la violencia que tienen los estudiantes de acuerdo a la siguiente tipología: violencia física: directa e indirecta, violencia verbal, violencia, social, disrupción en el aula, violencia a través de redes sociales y violencia de parte del profesor hacia el alumnado. Es importante mencionar que el test CUVE-3 no debe ser aplicado deliberadamente a cualquier edad, sino que debe de existir una adaptación 
adecuada a la edad correspondiente. En específico se presentan dos tipos de CUVE-3: EP y CUVE-ESO; el primero de ellos se puede aplicar a estudiantes de entre 10 y 13 años, mientras que el segundo se recomienda la utilización en educación secundaria, bachilleres y alumnos que tengan entre 13 y 19 años.

La prueba contaba con solo 29 Ítems, representadas en oraciones o situaciones dentro del aula, sin embargo, se agregaron 13 oraciones nuevas, por los nuevos aspectos de violencia que se presentan dentro del ámbito escolar, como lo son los insultos o acosos en redes sociales, la exclusión social o discriminación y la disrupción en el aula.

La forma de contestar el CUVE-R está organizada por numeración que van del 1 al 5, siendo 1 "nunca" hasta llegar al 5 como "siempre", a través de una escala tipo Likert.

Participantes: Se eligieron alumnos de $5^{\circ}$ y $6^{\circ}$ grado de una escuela primaria del estado de Chihuahua en, siendo 87 alumnos en total los que contestaron el test CUVE-3.

Figura 2. Grupos y número de participantes encuestados.

\begin{tabular}{ll} 
Grupo & Participantes \\
\hline $5^{\circ} " \mathrm{~A} "$ & 18 \\
$5^{\circ} " \mathrm{~B} "$ & 21 \\
$6^{\circ} \mathrm{A} "$ & 24 \\
$6^{\circ} \mathrm{B} "$ & 24 \\
\hline
\end{tabular}

Nota. Estudiantes que dieron respuesta a al test CUVE-R.

A cada alumno se le asignó un folio desde 5A1 hasta 6B87, para poder dar orden a las respuestas que los alumnos otorgaron. Se promedió los resultados de cada test resuelto, para obtener los datos de cada alumno, tomando como media 1.8709, siendo $53 \%$ los que rebasaron la media, en cuestión a la percepción de la violencia que manifiestan.

\section{Análisis de datos}

Análisis por grupo

Se optó por realizar gráficos, en las que se manifestaron los atípicos, o bien aquellos que no se encontraban en la normalidad del alumnado, que variaban en su respuesta, para poder obtener un referente visual de la situación de cada grupo. 
RECIE. Revista Electrónica Científica de Investigación Educativa Vol. 4, núm. 2, enero-diciembre 2019, pp. 1349-1360.

Figura 3. Grupo de $5^{\circ} \mathrm{A}$.

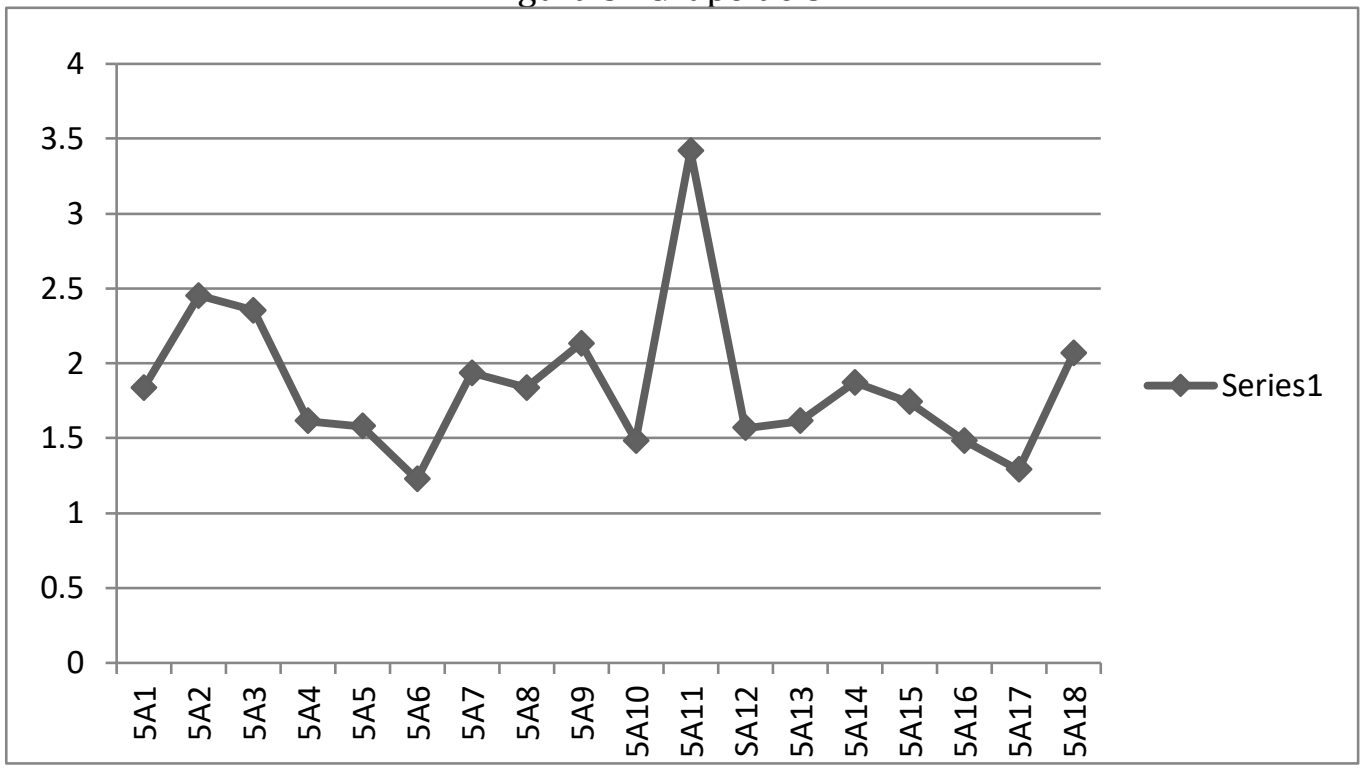

Figura 4. Grupo de $5^{\circ} \mathrm{B}$.

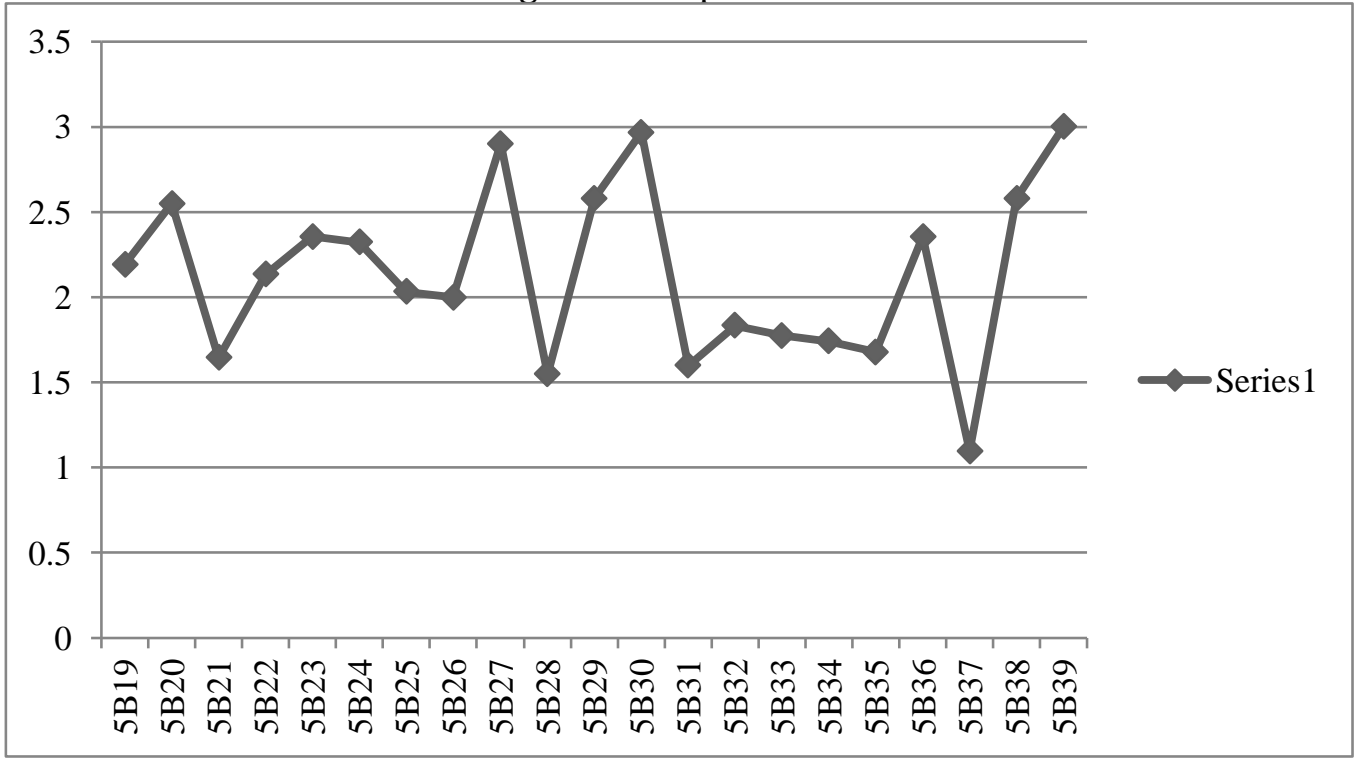

Sapién Zúñiga, L.F.; Ledezma Rivas, P.; y Ramos Trevizo, J.G. 
Figura 5. Grupo de $6^{\circ} \mathrm{A}$.

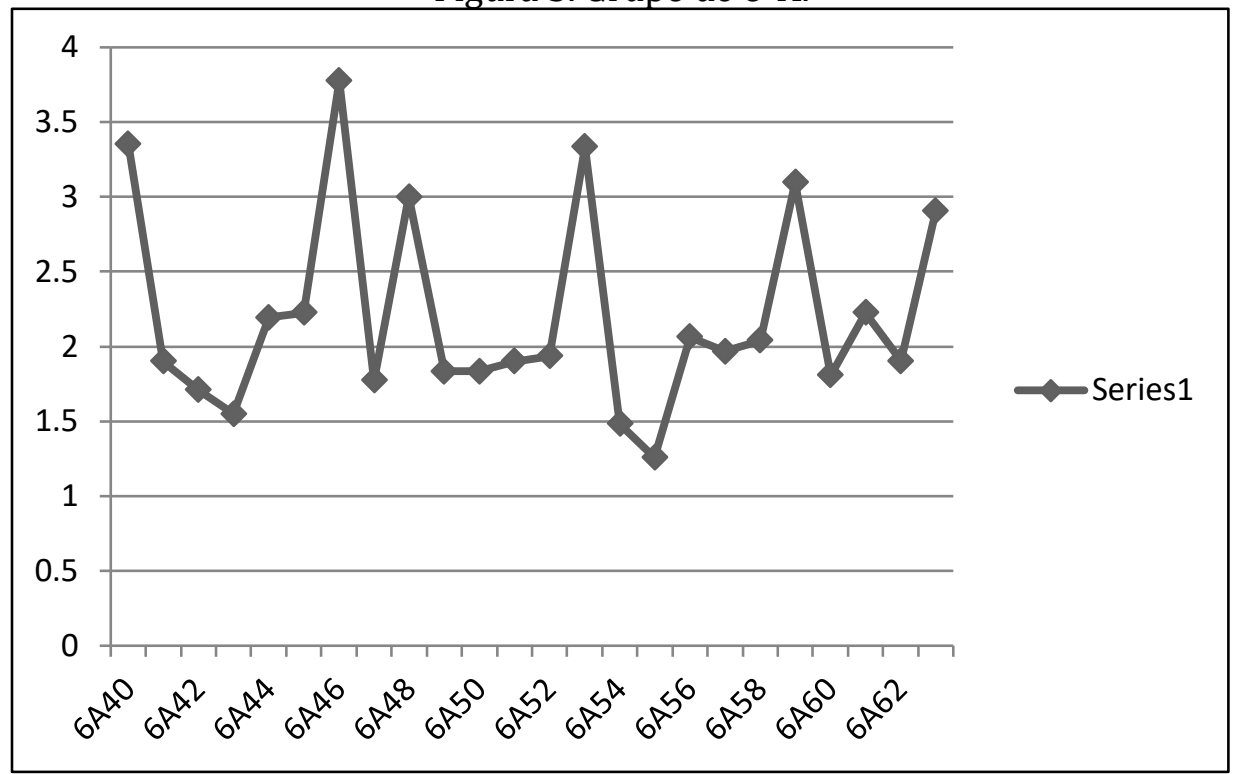

Figura 6. Grupo de 6 "'B".

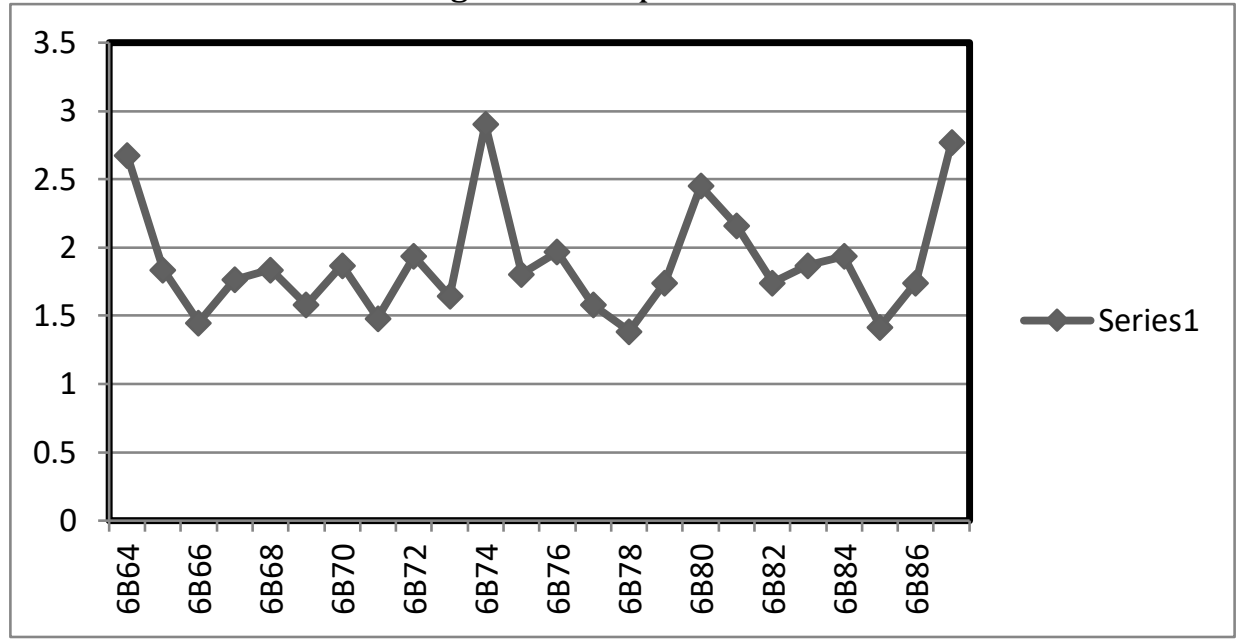

En general, los 4 grupos presentan de 1 hasta 6 variaciones importantes a analizar. Existen dos posibilidades latentes para aquellos alumnos que tienen niveles más bajos, en referencia al resto del grupo, siendo que no estén contestando con total honestidad, o bien que estén desinteresados de la problemática que se viva dentro de su aula. El grupo de $5^{\circ} \mathrm{B}$ y de $6^{\circ} \mathrm{A}$ son los grupos que perciben un nivel de violencia más detallado en su alumnado, siendo el grupo de $6^{\circ} \mathrm{A}$ quienes presentan 6 alumnos visualmente atípicos.

Percepción de la violencia escolar en el último ciclo de educación primaria 
RECIE. Revista Electrónica Científica de Investigación Educativa Vol. 4, núm. 2, enero-diciembre 2019, pp. 1349-1360.

El grupo de $6^{\circ} \mathrm{A}$ presenta una característica especial a diferencia de los demás grupos, ya que se mantienen en niveles más altos, el grupo en general, siendo tanto víctimas como victimarios de los distintos tipos de violencia. El 25\% de este grupo son aquellos que perciben más la violencia.

Pero aparte de reconocer que grupos está percibiendo más la violencia, es importante demostrar que tipo de violencia se presenta más, para ello, se debe considerar que cada ítem está enfocado en la distinta tipología de violencia presentada en el contexto escolar.

\begin{tabular}{|c|c|c|c|c|c|c|}
\hline Item & 1 & 2 & 3 & 4 & 5 & Tipo de violencia \\
\hline 1 & 9 & 16 & 24 & 20 & 17 & $\begin{array}{l}\text { Violencia verbal del alumnado } \\
\text { hacia el alumnado }\end{array}$ \\
\hline 2 & 31 & 27 & 17 & 10 & 2 & $\begin{array}{l}\text { Violencia física indirecta por parte } \\
\text { del alumnado }\end{array}$ \\
\hline 3 & 1 & 15 & 27 & 21 & 23 & $\begin{array}{l}\text { Violencia verbal del alumnado } \\
\text { hacia el alumnado }\end{array}$ \\
\hline 4 & 57 & 18 & 8 & 0 & 4 & $\begin{array}{l}\text { Exclusión social } \\
\text { Violencia de profesorado a }\end{array}$ \\
\hline 5 & 55 & 17 & 8 & 2 & 3 & $\begin{array}{l}\text { alumnado } \\
\text { Violencia a través de las }\end{array}$ \\
\hline 6 & 57 & 16 & 3 & 6 & 5 & $\begin{array}{l}\text { Tecnologías de la Información y } \\
\text { Comumicación }\end{array}$ \\
\hline 7 & 4 & 28 & 19 & 14 & 21 & $\begin{array}{l}\text { Violencia verbal del alumnado } \\
\text { hacia el alumnado }\end{array}$ \\
\hline 8 & 32 & 28 & 19 & 4 & 4 & $\begin{array}{l}\text { Exclusión social } \\
\text { Violencia del profesorado hacia el }\end{array}$ \\
\hline 9 & 66 & 10 & 7 & 3 & 0 & $\begin{array}{l}\text { alumnado } \\
\text { Violencia verbal del alumno al }\end{array}$ \\
\hline 10 & 46 & 23 & 15 & 0 & 3 & profesorado \\
\hline 11 & 12 & 27 & 19 & 22 & 6 & Disrupción en el aula \\
\hline 12 & 61 & 15 & 5 & 4 & 1 & $\begin{array}{l}\text { Violencia del profesorado hacia el } \\
\text { alumnado } \\
\text { Violencia a través de las } \\
\text { Tecnologías de la Información y }\end{array}$ \\
\hline 13 & 47 & 20 & 9 & 3 & 6 & $\begin{array}{l}\text { Comunicación } \\
\text { Violencia verbal del alumnado al }\end{array}$ \\
\hline 14 & 60 & 15 & 8 & 2 & 2 & $\begin{array}{l}\text { profesorado } \\
\text { Violencia del profesorado hacia el }\end{array}$ \\
\hline 15 & 52 & 17 & 6 & 7 & 2 & $\begin{array}{l}\text { alumnado } \\
\text { Violencia física indirecta por parte }\end{array}$ \\
\hline 16 & 69 & 8 & 6 & 1 & 1 & $\begin{array}{l}\text { del alumnado } \\
\text { Violencia verbal del alumnado }\end{array}$ \\
\hline 17 & 12 & 30 & 14 & 20 & 10 & hacia el alumnado \\
\hline 18 & 49 & 19 & 10 & 6 & 2 & $\begin{array}{l}\text { Violencia del profesorado hacia el } \\
\text { alumnado }\end{array}$ \\
\hline 19 & 17 & 23 & 15 & 14 & 18 & $\begin{array}{l}\text { Violencia física directa y } \\
\text { amenazas a estudiantes }\end{array}$ \\
\hline 20 & 76 & 7 & 2 & 0 & 1 & $\begin{array}{l}\text { Violencia a través de las } \\
\text { Tecnologías de la Información y } \\
\text { Comunicación }\end{array}$ \\
\hline 21 & 27 & 34 & 11 & 9 & 6 & $\begin{array}{l}\text { Violencia física directa y } \\
\text { amenazas entre estudiantes }\end{array}$ \\
\hline 22 & 9 & 28 & 24 & 11 & 15 & Disrupción en el aula \\
\hline 23 & 72 & 4 & 1 & 1 & 2 & $\begin{array}{l}\text { Violencia de profesorado hacia } \\
\text { alumnado }\end{array}$ \\
\hline
\end{tabular}

Figura 7. Frecuencia de ítems y tipo de violencia.

Nota. Se muestran la frecuencia de resultados por ítem, y el tipo de violencia al que corresponden. Construcción propia. 
De color rojo se pueden encontrar los resultados que tuvieron una mayor frecuencia de resultados, de manera general los resultados están en rangos aceptables, ya que son 21 ítems de 31 los que se encuentran en niveles mínimos, siendo la respuesta de "nunca" la predominante. Sin embargo, "Algunas veces" y "Casi nunca" son respuestas que también se presentan.

De color amarillo, dentro de la misma tabla, se encuentran subrayados los tipos de violencia más recurrentes, según los resultados del alumnado, siendo estos: violencia verbal del alumnado hacia el alumnado y disrupción en el aula con más frecuencia, y con menos frecuencia, pero de igual manera resaltante, se encuentra la violencia física directa y amenazas en estudiantes, y violencia indirecta por parte del alumnado.

\section{Conclusiones}

Al contrario de lo que se creía al iniciar la investigación, la violencia física aparece como una de las formas de agresión menos percibida en la escuela donde se realizó el estudio. Por otro lado, la violencia verbal entre los estudiantes está presente como la forma más frecuente de violencia según el CUVE. Es decir, hablar mal unos de otros, decir chismes, agredir con palabras altisonantes a los demás representa para los chicos del último ciclo escolar de educación primaria en la institución donde se realiza el estudio, una de las maneras más frecuentes de agresión.

La mayoría de los resultados se mantienen en niveles aceptables, es importante analizar que, aun así, hay alumnos que llegaron a contestar con el valor de "siempre", esto puede demostrar que, aunque la mayoría percibe poca violencia, existen alumnos que están manifestando estas situaciones, ya sea como víctimas o victimarios, percibiendo que la escuela no es exenta de la violencia.

Los tipos de violencia que se ven menos reflejados en la aplicación del CUVE-3 son la del profesorado al alumnado y la relacionada con las tecnologías de la información y comunicación, con esto se aprueban las relaciones que se mantienen del profesorado hacia los alumnos, en cuestión a trato justo y respetuoso.

La pretensión es pasar de la invisibilidad de algunos tipos de violencia para pasar a la acción en cuanto a generar insumos que permitan a los tomadores de decisiones generar espacios de convivencia armónicos y que potencien el desarrollo de las habilidades de todos y todas. 
RECIE. Revista Electrónica Científica de Investigación Educativa Vol. 4, núm. 2, enero-diciembre 2019, pp. 1349-1360.

\section{Referencias}

Cerezo, F. (2009). Bullying: análisis de la situación en las aulas españolas. International Journal of Psychology and Psychological Therapy, 9, 383-394..

Espín F., Valladares, A.; Abad, J, Clarivel. P., Gener, N. (2008). La violencia, un problema de salud. Scielo, 24, 1.

Etienne G., Linda L. Dahlberg, J., Mercy, A., Zwi y Rafael Lozano. (2003). Informe mundial sobre la violencia y la salud. enero 2018, de OMS Sitio web:

http://iris.paho.org/xmlui/bitstream/handle/123456789/725/927

5315884.pdf?sequence $=1$ \&isAllowed $=y$

Gómez Nashiki, A. (2005). Violencia e institución educativa. Revista Mexicana de Investigación Educativa, 10 (26), 693-718.

Grupo ALBOR-COHS. (2012). CUVE3 Manual de Referencia. Bizkaia, España: COHS

Monje, C.. (2011). Metodología de la investigación cuantitativa y cualitativa. Colombia: Programa de comunicación social y periodismo.

Olweus, D. (1996). Problemas de hostigamiento y de víctimas en la escuela. Perspectivas, vol. XXVI, 2, 357-389

Organización Mundial de la Salud. (1999). Informe mundial sobre la violencia y la salud. Ginebra: OMS.

Real Academia Española. (2007). Diccionario de la lengua española (23.aed.). Madrid, España

Schwartz, D., Proctor, L.J. y Chien, D.H. (2001). The aggressive victim of bullying: Emotional and behavioral dysregulation as a pathway to victimization by peers. En J. Juvonen y S. Graham (Eds), Peer harassment in school: The plight of the vulnerable and victimized (pp. 147-174). New York: Guilford Press. 